\title{
Entropy of Localized States and Black Hole Evaporation
}

\author{
Ken D. Olumit \\ Center for Theoretical Physics \\ Laboratory for Nuclear Science \\ and Department of Physics \\ Massachusetts Institute of Technology \\ Cambridge, Massachusetts 02139 \\ (MIT-CTP-2580, hep-th/9610086, November 1996)
}

\begin{abstract}
We call a state "vacuum-bounded" if every measurement performed outside a specified interior region gives the same result as in the vacuum. We compute the maximum entropy of a vacuum-bounded state with a given energy for a one-dimensional model, with the aid of numerical calculations on a lattice. The maximum entropy is larger than it would be for rigid wall boundary conditions by an amount $\delta S$, which for large energies is $\lesssim \frac{1}{6} \ln \left(L_{\text {in }} T\right)$, where $L_{\text {in }}$ is the length of the interior region. Assuming that the state resulting from the evaporation of a black hole is similar to a vacuum-bounded state, and that the similarity between vacuum-bounded and rigid-wall-bounded problems extends from 1 to 3 dimensions, we apply these results to the black hole information paradox. We conclude that large amounts of information cannot be emitted in the final explosion of a black hole.
\end{abstract}

Submitted to Phys. Rev. D.

Typeset using REVTEX

\footnotetext{
${ }^{\dagger}$ Email address: kdo@ctp.mit.edu

${ }^{*}$ This work is supported in part by funds provided by the U.S. Department of Energy (D.O.E.) under cooperative research agreement \#DF-FC02-94ER40818.
} 


\section{INTRODUCTION}

\section{A. Background}

Since the discovery of black hole radiation by Hawking [1], the fate of information falling into a black hole has been a mystery. (See [2,3,4] for reviews.) If Hawking's semiclassical calculation is correct, then the outgoing radiation is purely thermal and the outgoing photons are uncorrelated to each other and to the matter which formed the black hole. If the evaporation is complete, and if the thermal nature of the radiation persists throughout the evaporation, then the original information is lost. Thus if the black hole is formed from a quantum-mechanically pure state, there will nevertheless be a mixed state after the evaporation is complete. This is the position held by Hawking (e.g. see [5]), but it violates CPT and may lead to difficulties with energy conservation and causality [2, 6, [, 8,

If information is not lost in black hole evaporation, there are several possibilities. One is that the black hole does not evaporate completely, but instead produces one or more Planck-scale remnants (e.g. see [四). Another possibility is that the information disappears into a baby universe [9]. In this scenario the quantum-mechanical pure state is preserved, but parts of it are inaccessible to observation. It is also possible that the radiation is not really thermal, even at early times, because of a complementarity principle [10,11] or the inapplicability of the semiclassical approach [12, 13, 14, and thus that the information is encoded in subtle correlations in the radiation. In this case the black hole could act like a normal object with the entropy describing internal degrees of freedom. Some results from string theory 15,16] tend to confirm this view.

Even if the radiation is thermal and uncorrelated during most of the evaporation, there is no reason to believe that it remains thermal near the endpoint of the evaporation. The late-time radiation is presumably governed by an unknown theory of quantum gravity, and may well have correlations to the radiation emitted earlier. 1 However, it is generally believed that late-time radiation cannot resolve the information paradox [17,3], 目. The argument goes as follows: While the black hole is large, it is presumably radiating high-entropy thermal radiation. If the final explosion is to restore a pure state, it must radiate as much entropy 2 as was radiated in earlier times. However, by the time the black hole reaches the point where unknown physics could come into play, there is little energy remaining. To radiate a lot of information with little energy requires a long period of time, and thus the "final explosion" looks more like a long-lived remnant.

However, Wilczek [18] argues from a moving mirror model that a state with high entropy can nevertheless be purified with arbitrarily low energy cost. In certain ways, his model looks more like a remnant theory than a complete-evaporation theory, but it still appears to cast some doubt on the standard argument above.

\footnotetext{
${ }^{1}$ This is conceivable because at early times information in the outgoing radiation can be correlated with information in the ingoing negative-energy flux.

${ }^{2}$ Here and throughout this paper, "entropy" means fine-grained quantum-mechanical entropy.
} 
In any case, this argument requires bounding the entropy that can be contained in a particular region with a fixed energy. In the case of a region with reflecting walls, this is the question of finding the thermal state of quantum fields in a box. For a spherical box and a particular field theory the problem is easily solved. But with a region of complex shape, or where one wishes to make a statement intended to apply to all field theories, the situation is more complicated. Bekenstein [19,20] argues that such a universal bound exists, although Unruh and Wald [21] disagree.

Here we take a different approach. We consider only a single scalar field, but we use a weaker and, we hope, more physical condition on the results of the black hole evaporation. In the end, our results still support the claim that late-time radiation cannot restore the purity of the state of an evaporating black hole.

\section{B. The "vacuum-bounded state"}

We start by considering a black hole formed from a pure quantum-mechanical state of incoming matter. To avoid any possible complications of quantum gravity theory, we will look at the state produced after the black hole has completely evaporated [3]. Gravity should play no significant role in this state, since the energy density state should be small everywhere. 3 We can describe the final state as follows: At large distances from the position of the black hole (taken as the origin) there is outgoing Hawking radiation, which we are assuming to be thermal and uncorrelated to anything. Within some distance $R$ of the origin, there is some state of ordinary quantum fields that could have correlations with the radiation emitted earlier. The distance $R$ is the distance to which such information might have propagated after unknown physics came into play. Let us assume that Hawking's semiclassical calculation is good up to an energy scale $T_{\text {unk }}$. This temperature is reached when the black hole's mass is $M_{0}=1 /\left(8 \pi T_{\text {unk }}\right)$. If, after this, the rate of evaporation continues to match the Hawking calculation, 5 the black hole will evaporate in time $t \sim 10^{4} \mathrm{M}^{3} / \mathrm{g} \sim$ $1 /\left(g T_{\mathrm{unk}}^{3}\right)$, where $g$ is the effective number of degrees of freedom in the particles that can be radiated. (See [22].) So there is a sphere of radius

$$
R \sim \frac{1}{g T_{\mathrm{unk}}^{3}}
$$

which contains total energy

$$
E_{0}=\frac{1}{8 \pi T_{\mathrm{unk}}}
$$

\footnotetext{
${ }^{3}$ If instead there are Planck-scale concentrations of energy, then we would have a remnant theory, a possibility we are explicitly not considering here.

${ }^{4}$ We are working with units in which $c=G=\hbar=k_{B}=1$

${ }^{5}$ As opposed, for example, to slowing to nothing and leaving a remnant.
} 
in which the information could be contained. Taking, for example, $T_{\text {unk }}=10^{15} \mathrm{Gev} \sim$ $10^{-4} m_{\mathrm{pl}}$ and $g \sim 100$ we get

$$
\begin{aligned}
R & \sim 10^{10} l_{\mathrm{pl}} \sim 10^{-23} \mathrm{~cm} \\
E_{0} & \sim 10^{3} m_{\mathrm{pl}} \sim 10^{-2} \mathrm{~g} \sim 10^{19} \mathrm{erg} .
\end{aligned}
$$

Now we would like to answer the following question: How much entropy can be contained in a spherical region of radius $R$ with a total energy of $E_{0}$ ? To answer this question we have to specify what we mean by "contained in a region". As mentioned earlier, if we ask how much entropy can be contained in a spherical box of radius $R$ with perfectly reflecting walls, the question can be easily answered. However, the system with the box is not so closely akin to the system under discussion. For instance, inserting the reflecting walls into the system produces a divergent increase in the ground-state energy of the system. Furthermore, if we started with the vacuum in the whole system, and then introduced a spherical wall, we would produce a divergent geometric entropy [23].24]. A better description of our system is simply that it has thermal radiation outside radius $R$, and an unknown state of the quantum fields inside radius $R$, but no barrier or boundary at $R$.

To study such systems we will assume that the difference between the external Hawking radiation and an external vacuum is not important to considerations of entropy. We will study systems that have an arbitrary state inside $R$ but the vacuum outside $R$. To make this precise we will specify the problem as follows:

Let a "vacuum-bounded state" be a generalized state (i.e. density matrix) for which every operator composed of fields at points outside a specified interior region has the same expectation value as in the vacuum. What is the maximum entropy of such a state whose interior region is a sphere of radius $R$ and whose total average energy 8 is given by $\langle H\rangle=E_{0}$ ?

We expect to find that the answer to this question is similar to that of a box of radius $R$ with reflecting walls, with some small correction.

\section{GENERAL CONSIDERATIONS}

We will first consider a general system divided into an inside region ("part 1") and an outside region ("part 2"). We will say that a generalized state (i.e. density matrix)

\footnotetext{
${ }^{6}$ Another possibility is that $g$ diverges as $T \rightarrow m_{\mathrm{pl}}$. In this case the information can be radiated in a small number of particles of about the Planck mass, chosen from an infinite spectrum of such particles. This is effectively a remnant theory.

${ }^{7}$ If this approximation is bad we can always increase $R$ until the Hawking radiation outside $R$ has very low temperature.

${ }^{8}$ We cannot specify that any measurement of the energy must give a particular value. Such a state is necessarily static and thus cannot represent outgoing radiation.
} 
is "localized to the inside" or "obeys the vacuum-bounded condition" if any measurement performed on the outside field operators in this state yields the same result as in the vacuum, i.e. if

$$
\operatorname{Tr} \rho O_{2}=\operatorname{Tr} \rho^{\mathrm{vac}} O_{2}=\left\langle 0\left|O_{2}\right| 0\right\rangle
$$

for every operator $O_{2}$ that is constructed out of field operators in the outside region.

In the language of density matrices, we can write $\rho_{2}=\operatorname{Tr}_{1} \rho$, where $\rho$ is the overall density matrix describing our system and $\operatorname{Tr}_{1}$ means to trace over all the "inside" variables. Then $\rho_{2}$ is the reduced density matrix describing only the outside variables. Eq. (2.1) then is equivalent to

$$
\rho_{2}=\rho_{2}^{\mathrm{vac}} \equiv \operatorname{Tr}_{1}|0\rangle\langle 0|,
$$

where $|0\rangle$ denotes the ground state.

\section{A. Stationary points of $S$}

The present problem is a particular case of the following general problem: Find a density matrix $\rho$ (i.e. a positive-semidefinite Hermitian matrix with unit trace) which maximizes $S=-\operatorname{Tr} \rho \ln \rho$ subject to a set of constraints $\operatorname{Tr} \rho C_{\alpha}=V_{\alpha}$. To analyze this problem we will look at the change in $S\left(\rho^{\prime}\right)$ when $\rho$ is varied via $\rho^{\prime}=\rho+t \delta \rho$. The naive result holds for the first derivative,

$$
\frac{d S}{d t}=-\operatorname{Tr}(\delta \rho \ln \rho+\delta \rho) .
$$

We will only consider variations of $\rho$ which leave $\rho$ normalized with $\operatorname{Tr} \rho=1$, which means that $\operatorname{Tr} \delta \rho=0$ and thus

$$
\frac{d S}{d t}=-\operatorname{Tr} \delta \rho \ln \rho .
$$

We show in appendix A that entropy is always strictly (downward) concave [25] which means that

$$
\frac{d^{2} S}{d t^{2}}<0
$$

for any $\delta \rho$. Thus any point at which $S$ is stationary is a local maximum. Furthermore, we can show that there can be at most one such maximum, as follows.

Let $\mathcal{P}$ be the space of all Hermitian matrices such that $\operatorname{Tr} \rho=1$ and $\operatorname{Tr} \rho C_{\alpha}=V_{\alpha}$, and let $\mathcal{P}_{+}$be the subspace of $\mathcal{P}$ where $\rho$ is positive-semidefinite. First we note that $\mathcal{P}_{+}$is convex: Let $\rho_{1}$ and $\rho_{2}$ be elements of $\mathcal{P}_{+}$and let $\rho(t)=t \rho_{1}+(1-t) \rho_{2}, t \in[0,1]$. The matrix $\rho(t)$ is clearly Hermitian, properly normalized, and satisfies the constraints. It is also positive semidefinite, since for any $\mathbf{x}, \mathbf{x} \cdot \rho(t) \mathbf{x}=t\left(\mathbf{x} \cdot \rho_{1} \mathbf{x}\right)+(1-t)\left(\mathbf{x} \cdot \rho_{2} \mathbf{x}\right) \geq 0$. Thus $\rho(t)$ is an admissible density matrix as desired. 
Since $S$ is a strictly concave function defined on a convex space, it cannot have more than one stationary point. For if we had two stationary points we could vary $\rho$ along a line between them, and since $d^{2} S / d t^{2}<0$ we could not have $d S / d t=0$ at both.

If there is any $\rho$ which achieves the maximum value of $S$, it must be a stationary point of $S$. The only conceivable alternative would be for $\rho$ to lie on the edge of $\mathcal{P}_{+}$in $\mathcal{P}$, but that is excluded by the following argument: Suppose $\rho$ is on the edge of $\mathcal{P}_{+}$in $\mathcal{P}$. Then there is some direction $\delta \rho$ such that $\rho^{\prime} \equiv \rho+t \delta \rho$ is positive-semidefinite for arbitrarily small positive $t$ but not for negative $t$ arbitrarily close to zero. That can happen only if $\rho$ has arbitrarily small or zero eigenvalues $\rho_{i}$ which become negative as $t$ becomes negative. For this to happen these eigenvalues must increase from arbitrarily small values as $t$ becomes positive. As $t \rightarrow 0$ these eigenvalues dominate everything else in $d S / d t=\sum_{i}\left(-\ln \rho_{i}\right)$, so $d S / d t \rightarrow+\infty$. Thus for sufficiently small but positive $t, S\left(\rho^{\prime}\right)>S(\rho)$, so $\rho$ does not maximize $S$.

Whether a maximum-entropy point exists at all depends on the constraints $C_{\alpha}$. First suppose there is a constraint on the energy $\operatorname{Tr} \rho H=E_{0}$ and no other constraints. In a normal system there will be a state with the maximum entropy 9 Now suppose that there are additional constraints. They further restrict the space of allowable $\rho$ and thus can only reduce the achievable values of $S$, so there is at least a supremum of possible values of $S$ in the constrained system. We believe but have not been able to prove that in our problem there will be a $\rho$ which achieves the maximum value of $S$. In the numerical work described in Sec. VII we have always succeeded in finding a solution.

\section{B. The form of $\rho$}

We must find the unique state $\rho$ which satisfies our constraints and which gives $\operatorname{Tr} \delta \rho \ln \rho=0$ for any $\delta \rho$ which maintains the constraints. This means that we are concerned with $\delta \rho$ such that

$$
\begin{array}{r}
\operatorname{Tr} \delta \rho=0 \\
\operatorname{Tr} \delta \rho C_{\alpha}=0
\end{array}
$$

for all $\alpha$.

Now we can treat the space of Hermitian operators as an inner product space with $(A, B) \equiv \operatorname{Tr} A B$. In this language, Eqs. (2.6) imply that the possible $\delta \rho$ span the space orthogonal to the identity and to each of the $C_{\alpha}$. Since $\ln \rho$ must be orthogonal to all the $\delta \rho$, it must be composed only of $I$ and the $C_{\alpha}$, so we can write

$$
\ln \rho=\text { const }+\sum_{\alpha} f_{\alpha} C_{\alpha}
$$

for some coefficients $f_{\alpha}$. Since $\operatorname{Tr} \rho=1$, we can write

\footnotetext{
${ }^{9}$ But note that if the density of states rises exponentially or faster with energy, then the entropy can be unbounded.
} 


$$
\rho=\frac{e^{\sum f_{\alpha} C_{\alpha}}}{\operatorname{Tr} e^{\sum f_{\alpha} C_{\alpha}}} .
$$

Our goal is now to determine the coefficients $f_{\alpha}$ so that the constraints are satisfied.

We can define a grand partition function,

$$
Q=\operatorname{Tr} e^{\sum f_{\alpha} C_{\alpha}}
$$

Its derivatives are

$$
\frac{d Q}{d f_{\alpha}}=\operatorname{Tr} C_{\alpha} e^{\sum f_{\alpha} C_{\alpha}}
$$

So

$$
\left\langle C_{\alpha}\right\rangle=\frac{d}{d f_{\alpha}} \ln Q
$$

We have the usual thermodynamic formula for the entropy,

$$
S=-\langle\ln \rho\rangle=\ln Q-\sum f_{\alpha}\left\langle C_{\alpha}\right\rangle .
$$

Differentiating this we find

$$
\frac{d S}{d f_{\alpha}}=-\sum f_{\beta} \frac{d\left\langle C_{\beta}\right\rangle}{d f_{\alpha}}
$$

Now we specialize to the case where one of the constraints is just the Hamiltonian. The corresponding coefficient is written $-\beta$, and we have

$$
\rho=\frac{1}{Q} e^{-\beta H+\sum f_{\alpha} C_{\alpha}} .
$$

If we vary the coefficients in such a way that $\langle H\rangle=E$ changes but the other $\left\langle C_{\alpha}\right\rangle=V_{\alpha}$ remain fixed we see that

$$
d S=-\sum f_{\alpha} d\left\langle C_{\alpha}\right\rangle=\beta d E .
$$

Thus $\beta=d S / d E$ and so the coefficient $\beta$ has the usual interpretation as the inverse temperature.

\section{ONE SCALAR FIELD, ONE DIMENSION.}

Now we will restrict ourselves to a theory consisting only of gravity and one massless scalar field. Presumably such a system has enough richness to contain the usual black hole information paradox, so nothing important is lost by making this reduction. We will also begin here by working in one dimension. We will put our entire system in a box of length $L$ and require that all deviations from the vacuum are in the region from 0 to $L_{\text {in }}$. Later we will let the overall box size $L$ go to infinity while $L_{\text {in }}$ remains fixed. The inside region will be $\left[0, L_{\text {in }}\right]$ and the outside region will be $\left[L_{\text {in }}, L\right]$. We will use the usual scalar field Hamiltonian, which in classical form is

$$
H=\frac{1}{2} \int_{0}^{L}\left[\pi(x)^{2}+\left(\frac{d \phi}{d x}\right)^{2}\right] d x
$$




\section{THE SOLUTION DENSITY MATRIX IS GAUSSIAN}

In our problem, the constraints are the energy bound,

$$
\operatorname{Tr} \rho H=E_{0},
$$

and the "vacuum-bounded" condition,

$$
\operatorname{Tr} \rho O_{2}^{\alpha}=\left\langle 0\left|O_{2}^{\alpha}\right| 0\right\rangle
$$

where $O_{2}^{\alpha}$ is any operator which is constructed out of the fields $\phi(x)$ and $\pi(x)$ in the outside region. The solution must have the form

$$
\rho \propto e^{-\beta H+\sum f_{\alpha} O_{2}^{\alpha}}
$$

We now show that $f_{\alpha}$ is nonzero only for those operators $O_{2}^{\alpha}$ which are quadratic in the fields.

Suppose that we wanted to solve a different problem in which we cared only about the constraints involving the quadratic operators. We would have the energy bound and the constraints

$$
\operatorname{Tr} \rho Q_{2}^{a}=\left\langle 0\left|Q_{2}^{a}\right| 0\right\rangle,
$$

where $Q_{2}^{a}$ runs only over quadratic operators $\phi(x) \phi(y)$ and $\pi(x) \pi(y)$. (The operators $\phi(x) \pi(y)$ vanish automatically by symmetry under $\phi \rightarrow-\phi$.) The solution to this problem has the form

$$
\rho^{\prime} \propto e^{-\beta H+\sum f_{a} Q_{2}^{\alpha}}
$$

Now $\rho^{\prime}$ is a Gaussian operator, i.e. $\left\langle\phi(\cdot)\left|\rho^{\prime}\right| \phi^{\prime}(\cdot)\right\rangle$ is a Gaussian functional of the values of $\phi$ and $\phi^{\prime}$. Let $\rho_{2}^{\prime}=\operatorname{Tr}_{1} \rho^{\prime}$. The trace is just a set of Gaussian integrals, which means that the resulting $\rho_{2}^{\prime}$ is also a Gaussian. Because $H$ is quadratic, the vacuum $\rho^{\text {vac }}=|0\rangle\langle 0|$ is Gaussian, and so is its trace $\rho_{2}^{\mathrm{vac}}=\operatorname{Tr}_{1} \rho^{\mathrm{vac}}$. Now by construction we have $\operatorname{Tr} \rho_{2}^{\prime} \phi(x) \phi(y)=$ $\operatorname{Tr} \rho_{2}^{\mathrm{vac}} \phi(x) \phi(y)$ and $\operatorname{Tr} \rho_{2}^{\prime} \pi(x) \pi(y)=\operatorname{Tr} \rho_{2}^{\mathrm{vac}} \pi(x) \pi(y)$. These conditions are sufficient to fix the coefficients in the Gaussian $\rho_{2}^{\prime}$, and thus to show that in fact $\rho_{2}^{\prime}$ and $\rho_{2}^{\text {vac }}$ are the same Gaussian, i.e. that $\operatorname{Tr}_{1} \rho^{\prime}=\operatorname{Tr}_{1} \rho^{\text {vac }}$.

Thus $\rho^{\prime}$ satisfies all the constraints of the original problem. Since only one $\rho$ can have these properties it follows that $\rho=\rho^{\prime}$ and thus that Gaussian solution $\rho^{\prime}$ is the correct solution to the original problem.

\section{THE DISCRETE CASE}

We now approximate the continuum by a one-dimensional lattice of coupled oscillators, with a classical Hamiltonian

$$
H=\frac{1}{2}\left(\mathbf{P}_{x} \cdot \mathbf{P}_{x}+\mathbf{x} \cdot K \mathbf{x}\right) .
$$


The simple kinetic term in Eq. (5.1) corresponds to choosing oscillators of unit mass, regardless of how densely they are packed. In terms of scalar-field variables this means that $x_{i}=\sqrt{a} \phi_{i}$ and $P_{i}=\pi_{i} / \sqrt{a}$ where $a=L /(N+1)$ is the lattice spacing, $\phi_{i}$ is the average of $\phi(x)$ over an interval of length $a$, and $\pi_{i}$ is the total momentum $\pi(x)$ in the interval.

The matrix $K$ gives the couplings between the oscillators and represents the $d \phi / d x$ term in the scalar field Hamiltonian. To approximate the continuum with the zero-field boundary condition we will imagine that we have $N$ oscillators located at the points $1 / N+1 \ldots N / N+1$. and the end oscillators are coupled to fixed-zero oscillators at 0 and 1 . Then

$$
K=\left(\begin{array}{ccccc}
2 g & -g & 0 & 0 & . \\
-g & 2 g & -g & 0 & . \\
0 & -g & . & . & . \\
0 & 0 & . & . & . \\
. & . & . & . & .
\end{array}\right)
$$

where $g=(N+1)^{2} / L^{2}$.

We are trying to satisfy the constraints

$$
\begin{aligned}
\operatorname{Tr} \rho H & =E_{0} \\
\operatorname{Tr} \rho x_{i} x_{j} & =\left\langle 0\left|x_{i} x_{j}\right| 0\right\rangle \\
\operatorname{Tr} \rho P_{i} P_{j} & =\left\langle 0\left|P_{i} P_{j}\right| 0\right\rangle,
\end{aligned}
$$

where $i$ and $j$ run over the oscillators which represent the outside region. We have shown that the solution will have the form

$$
\rho \propto e^{-\beta H+f_{\mu \nu}^{(x)} x_{\mu} x_{\nu}+f_{\mu \nu}^{(P)} P_{\mu} P_{\nu}} .
$$

We can write this in a more familiar form as

$$
\rho \propto e^{-\beta H^{\prime}},
$$

where $H^{\prime}$ is a fictitious Hamiltonian for these oscillators,

$$
H^{\prime}=\frac{1}{2}\left(\mathbf{P}_{x} \cdot T^{\prime} \mathbf{P}_{x}+\mathbf{x} \cdot K^{\prime} \mathbf{x}\right)
$$

with

$$
T^{\prime}=\left(\begin{array}{c|c}
I & 0 \\
\hline 0 & T_{22}^{\prime}
\end{array}\right)
$$

and

$$
K^{\prime}=\left(\begin{array}{l|l}
K_{11} & K_{12} \\
\hline K_{12}^{T} & K_{22}^{\prime}
\end{array}\right)
$$

Here $K_{11}$ and $K_{12}$ are the sections of the original coupling matrix $K$, and $T_{22}^{\prime}$ and $K_{22}^{\prime}$ and $\beta$ can be adjusted in an attempt to meet the necessary conditions. This gives us one number, $\beta$, and two symmetric $N_{\text {out }}$-by- $N_{\text {out }}$ matrices, $K_{22}^{\prime}$ and $T_{22}^{\prime}$, that we can adjust. The constraints involve one scalar constraint, for $H$, and two $N_{\text {out }}$-by- $N_{\text {out }}$ symmetric matrices of constraints, for $x_{i} x_{j}$ and $P_{i} P_{j}$. There are equal numbers of equations to satisfy and free parameters to adjust, and so, if we are lucky, we will be able to find a solution. If we do find a solution, we know it is unique from the arguments of Sec. ПIA. 


\section{A. Computing the expectation values}

To actually solve these equations we will need to compute the expectation values of $x_{i} x_{j}$ and $P_{i} P_{j}$ given the density matrix $\rho \propto \exp \left\{-\beta H^{\prime}\right\}$. We can compute them in the usual way as derivatives of the partition function

$$
Q=\operatorname{Tr} e^{-\beta \cdot \frac{1}{2}\left(K_{\mu \nu}^{\prime} x_{\mu} x_{\nu}+T_{\mu \nu}^{\prime} P_{\mu} P_{\nu}\right)},
$$

so

$$
\begin{aligned}
& \left\langle x_{i} x_{j}\right\rangle=-\frac{1}{\beta}\left(\frac{d}{d K_{i j}^{\prime}}+\frac{d}{d K_{j i}^{\prime}}\right) \ln Q \\
& \left\langle P_{i} P_{j}\right\rangle=-\frac{1}{\beta}\left(\frac{d}{d T_{i j}^{\prime}}+\frac{d}{d T_{j i}^{\prime}}\right) \ln Q .
\end{aligned}
$$

To compute the partition function we will first find the normal modes of the fictitious classical problem with this Hamiltonian, and then treat these as independent oscillators which we will quantize. The Hamiltonian $H^{\prime}$ gives rise to the equations of motion

$$
\frac{d^{2} x_{\mu}}{d t^{2}}=-T_{\mu \nu}^{\prime} K_{\nu \lambda}^{\prime} x_{\lambda},
$$

so we look for eigenvectors $\mathbf{x}^{\alpha}$ that satisfy

$$
T^{\prime} K^{\prime} \mathbf{x}^{\alpha}=\omega_{\alpha}^{2} \mathbf{x}^{\alpha} .
$$

The eigenvectors will be complete, so that we can define new coordinates $z_{\alpha}$ via $\mathbf{x}=\sum z_{\alpha} \mathbf{x}^{\alpha}$, which will then obey the equations of motion

$$
\frac{d^{2} z_{\alpha}}{d t^{2}}=-\omega_{\alpha}^{2} z_{\alpha} .
$$

We can choose the norms of the eigenvectors so that $\mathbf{x}^{\alpha} \cdot K^{\prime} \mathbf{x}^{\beta}=\omega_{\alpha}^{2} \delta_{\alpha \beta}$ and group the eigenvectors into a matrix $V$ via $V_{\mu \alpha}=x_{\mu}^{\alpha}$. Then we will find that $T_{\alpha \beta}^{\prime}=x_{\mu}^{\alpha} x_{\mu}^{\beta}$, or

$$
K^{\prime}=V^{-1^{T}} \Omega^{2} V^{-1} \quad \text { and } \quad T^{\prime}=V V^{T}
$$

where $\Omega_{\alpha \beta}=\omega_{\alpha} \delta_{\alpha \beta}$. We can then substitute

$$
\mathbf{x}=V \mathbf{z} \quad \mathbf{P}_{x}=V^{-1^{T}} \mathbf{P}_{z}
$$

into $H^{\prime}$ to get

$$
\begin{aligned}
H^{\prime} & =\frac{1}{2}\left(\mathbf{P}_{z} \cdot \mathbf{P}_{z}+\mathbf{z} \cdot \Omega^{2} \mathbf{z}\right) \\
& =\frac{1}{2} \sum_{i}\left(P_{\alpha}^{2}+\Omega_{\alpha}^{2} z_{\alpha}^{2}\right) \equiv \sum_{\alpha} H_{\alpha}^{\prime} .
\end{aligned}
$$


This is the Hamiltonian for a set of (fictitious) uncoupled oscillators with frequencies $\omega_{i}$, and thus $Q=\operatorname{Tr} e^{-\beta H^{\prime}}$ will be the product of partition functions $Q_{\alpha}=\operatorname{Tr} e^{-\beta H_{\alpha}^{\prime}}$ for the individual oscillators. The partition function of a single oscillator is easily found to be

$$
Q_{i}=\frac{e^{-\beta \omega_{\alpha} / 2}}{1-e^{-\beta \omega_{\alpha}}}=\frac{1}{2} \operatorname{csch} \frac{\beta}{2} \omega
$$

and thus

$$
\ln Q=\sum \ln Q_{\alpha}=-\sum \ln \left(2 \sinh \frac{\beta}{2} \omega_{\alpha}\right) .
$$

Differentiating gives

$$
\frac{d}{d \omega_{\alpha}} \ln Q=-\frac{\beta}{2} \operatorname{coth} \frac{\beta}{2} \omega_{\alpha} .
$$

Letting $\Lambda_{\alpha}=\omega_{\alpha}^{2}$, this becomes

$$
\frac{d}{d \Lambda_{\alpha}} \ln Q=-\frac{\beta}{4 \omega_{\alpha}} \operatorname{coth} \frac{\beta}{2} \omega_{\alpha} .
$$

We can compute the effect of a change to $K_{i j}^{\prime}$ or $T_{i j}^{\prime}$ on the eigenvalues,

$$
\frac{d \Lambda_{\alpha}}{d K_{i j}^{\prime}}=V_{i \alpha} V_{j \alpha}
$$

and

$$
\frac{d \Lambda_{\alpha}}{d T_{i j}^{\prime}}=V_{\alpha i}^{-1} V_{\alpha j}^{-1} \Lambda_{\alpha}
$$

Finally,

$$
\begin{gathered}
\left\langle x_{i} x_{j}\right\rangle=\sum_{\alpha} \frac{1}{2 \omega_{\alpha}} V_{i \alpha} V_{j \alpha} \operatorname{coth} \frac{\beta}{2} \omega_{\alpha} \\
\left\langle P_{i} P_{j}\right\rangle=\sum_{\alpha} \frac{\omega_{\alpha}}{2} V_{\alpha i}^{-1} V_{\alpha j}^{-1} \operatorname{coth} \frac{\beta}{2} \omega_{\alpha} .
\end{gathered}
$$

These expressions must have the same values when $V$ and the $\omega$ are the eigenvectors and frequencies of $T^{\prime} K^{\prime}$ as they have in the vacuum, i.e. when $V$ and $\omega$ are the normal modes and frequencies of the vacuum and $\beta=\infty$.

\section{THE FORM OF THE NORMAL MODES}

We are trying to find the normal mode eigenvectors $\mathbf{x}^{\alpha}$ and frequencies $\omega_{\alpha}$ that will satisfy our constraints. If we take the $\omega_{\alpha}$ as given, we can learn about the $\mathbf{x}^{\alpha}$ as follows: The vectors $\mathbf{x}^{\alpha}$ satisfy the equation 


$$
T^{\prime} K^{\prime} \mathbf{x}^{\alpha}=\omega_{\alpha}^{2} \mathbf{x}^{\alpha}
$$

with

$$
T^{\prime}=\left(\begin{array}{c|c}
I & 0 \\
\hline 0 & T_{22}^{\prime}
\end{array}\right)
$$

and

$$
K^{\prime}=\left(\begin{array}{c|c|c}
\multicolumn{2}{c|}{K_{11}} & \multicolumn{2}{|c}{0} \\
\cline { 2 - 2 } & -g & -g \\
\hline 0 & K_{22}^{\prime}
\end{array}\right) .
$$

Let us introduce the convention that Latin letters from the start of the alphabet, $a, b, c, \ldots$ range over only "inside" oscillator indices, Latin letters from the middle of the alphabet, $i, j, k, \ldots$ range over only "outside" oscillator indices, and Greek letters range over all indices. Writing the eigenvalue equation out in components,

$$
T_{\mu \nu}^{\prime} K_{\nu \lambda}^{\prime} x_{\lambda}^{\alpha}=\omega_{\alpha}^{2} x_{\mu}^{\alpha} .
$$

Taking only the inside components of the eigenvalue equation, we see that

$$
K_{a \mu}^{\prime} x_{\mu}^{\alpha}=\omega_{\alpha}^{2} x_{a}^{\alpha} .
$$

That is to say,

$$
\begin{aligned}
2 g x_{1}^{\alpha}-g x_{2}^{\alpha} & =\omega_{\alpha}^{2} x_{1}^{\alpha} \\
-g x_{1}^{\alpha}+2 g x_{2}^{\alpha}-g x_{3}^{\alpha} & =\omega_{\alpha}^{2} x_{2}^{\alpha} \\
\cdots & \\
-g x_{N_{\text {in }}-1}^{\alpha}+2 g x_{N_{\text {in }}}^{\alpha}-g x_{N_{\text {in }}+1}^{\alpha} & =\omega_{\alpha}^{2} x_{N_{\text {in }}}^{\alpha} .
\end{aligned}
$$

Taking $\omega_{\alpha}$ fixed, there are $N_{\text {in }}$ equations involving $N_{\text {in }}+1$ unknown components of $\mathbf{x}^{\alpha}$. However, the equations are invariant under a uniform rescaling of $\mathbf{x}^{\alpha}$. Thus these equations fix $x_{\mu}^{\alpha}$ for $\mu=1 \ldots N_{\text {in }}+1$, except for normalization. The equations are readily solved, and the solution is

$$
x_{\mu}^{\alpha}=N_{\alpha}^{\prime} \sin \mu k_{\alpha}^{\prime},
$$

where

$$
\cos k_{\alpha}^{\prime}=1-\frac{\omega_{\alpha}^{2}}{2 g},
$$

and $N_{\alpha}^{\prime}$ is an unknown normalization factor. Here $k_{\alpha}^{\prime}$ and $N^{\prime \alpha}$ can be complex, but $\mathbf{x}^{\alpha}$ must be real. When $k_{\alpha}^{\prime}$ is real we will write $k_{\alpha}=k_{\alpha}^{\prime}$ and $N_{\alpha}=N_{\alpha}^{\prime}$ and call this a "normal" mode. When $k_{\alpha}^{\prime}$ is complex we can write 


$$
\begin{aligned}
k_{\alpha}^{\prime} & =\pi+i k_{\alpha} \\
N_{\alpha}^{\prime} & =i N_{\alpha} \\
x_{\mu}^{\alpha} & =(-)^{\mu-1} N_{\alpha} \sinh \mu k_{\alpha}
\end{aligned}
$$

with $k_{\alpha}$ and $N_{\alpha}$ real. We will refer to these as "abnormal" modes.

As before, we group the eigenvalues into a matrix, $V_{\mu \alpha}=x_{\mu}^{\alpha}$ and arrange the normalizations so that $K^{\prime}=V^{-1^{T}} \Lambda V^{-1}$ and $T^{\prime}=V V^{T}$. We know the value of $T^{\prime}$ if one of its indices is an inside index,

$$
\delta_{a b}=T_{a b}=V_{a \alpha} V_{b \alpha}
$$

and

$$
0=T_{a j}=V_{a \alpha} V_{j \alpha}
$$

From Eqs. (6.7) and (6.10) we get

$$
\delta_{a b}=\sum_{\alpha} N_{\alpha}^{\prime 2} \sin k_{\alpha}^{\prime} a \sin k_{\alpha}^{\prime} b .
$$

Since $V_{N_{\text {in }}+1, \alpha}=N^{\prime} \sin k_{\alpha}^{\prime}\left(N_{\text {in }}+1\right)$, we can use Eq. (6.11) to extend the range of Eq. (6.12) to $1 \ldots N_{\text {in }}+1$ except that the equation does not hold when both $a$ and $b$ have this value.

A similar calculation can be done for $V^{-1}$, the inverse of the eigenvalue matrix $V$. In this case we will find that

$$
V_{\alpha a}^{-1}=N_{\alpha}^{\prime} \sin k_{\alpha}^{\prime} a=V_{a \alpha} .
$$

This has the same form as the equation for $V$, but applies only for $a=1 \ldots N_{\text {in }}$. That makes $V^{-1}$ less useful than $V$ for establishing a connection between the inside and the outside region, and we will not use it further.

\section{NUMERICAL STUDIES}

We have solved numerically the set of nonlinear equations, Eqs. (5.23), with $\beta$ fixed and various values of $L_{\text {in }}$ and $N$. The problem is one of solving $N_{\text {out }}\left(N_{\text {out }}+1\right)$ simultaneous nonlinear equations for $N_{\text {out }}\left(N_{\text {out }}+1\right)$ parameters. In general such problems are quite difficult to solve, even if we know that there is a unique solution. Here we used the Powell hybrid method [26]. If there are no local minima of the rms error in the function values, this method converges from any starting point. Fortunately this appears to be the case in our problem. However, Powell's method often converges quite slowly for large systems, requiring many thousands of iterations to make progress. This has limited our numerical solutions to problems with no more than about 30 oscillators. The codes were written in Lisp and executed on DEC Alpha workstations. The calculations were done using an arbitraryprecision floating point package. The results presented here were computed using at least 38 digits of precision and the solutions found were accurate to at least 21 significant digits.

To understand the numerical solution we look at the normal mode frequencies and the forms of the normal modes. When the mode is "normal" (i.e. real $k_{\alpha}^{\prime}$ in Eq. (6.7)) the mode 


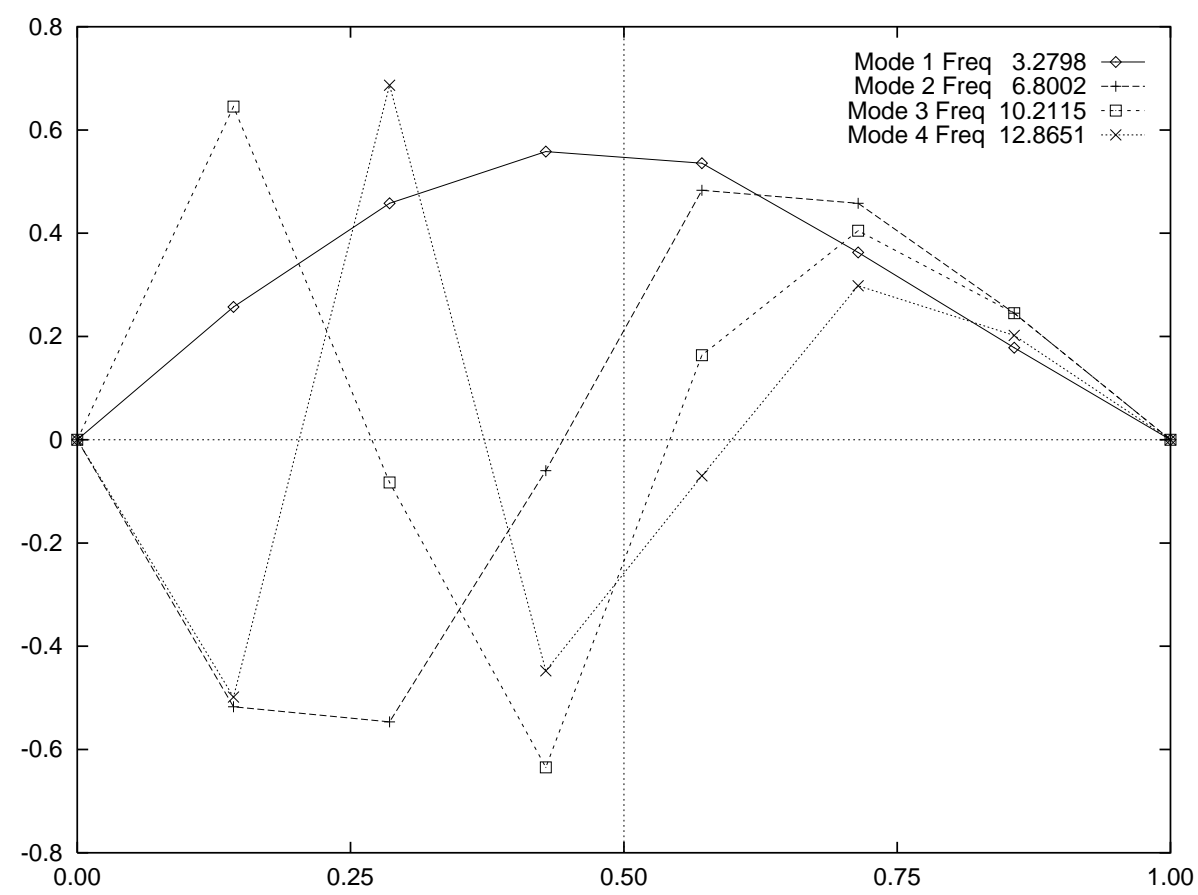

FIG. 1. Modes and frequencies for the "normal" modes of a system with $L=1.0, L_{\text {in }}=0.5$, $\beta=2, N=6$.

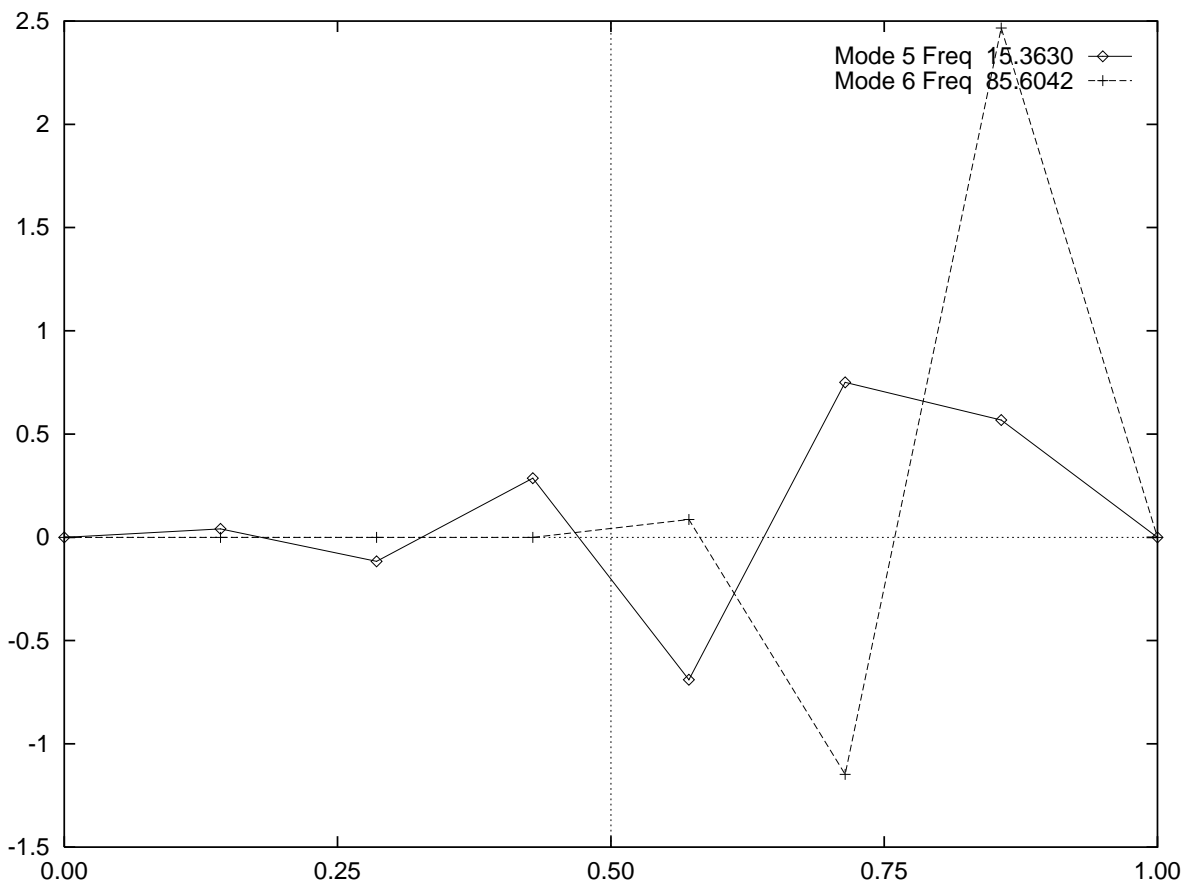

FIG. 2. Modes and frequencies for the "abnormal" modes of a system with $L=1.0, L_{\text {in }}=0.5$, $\beta=2, N=6$. 


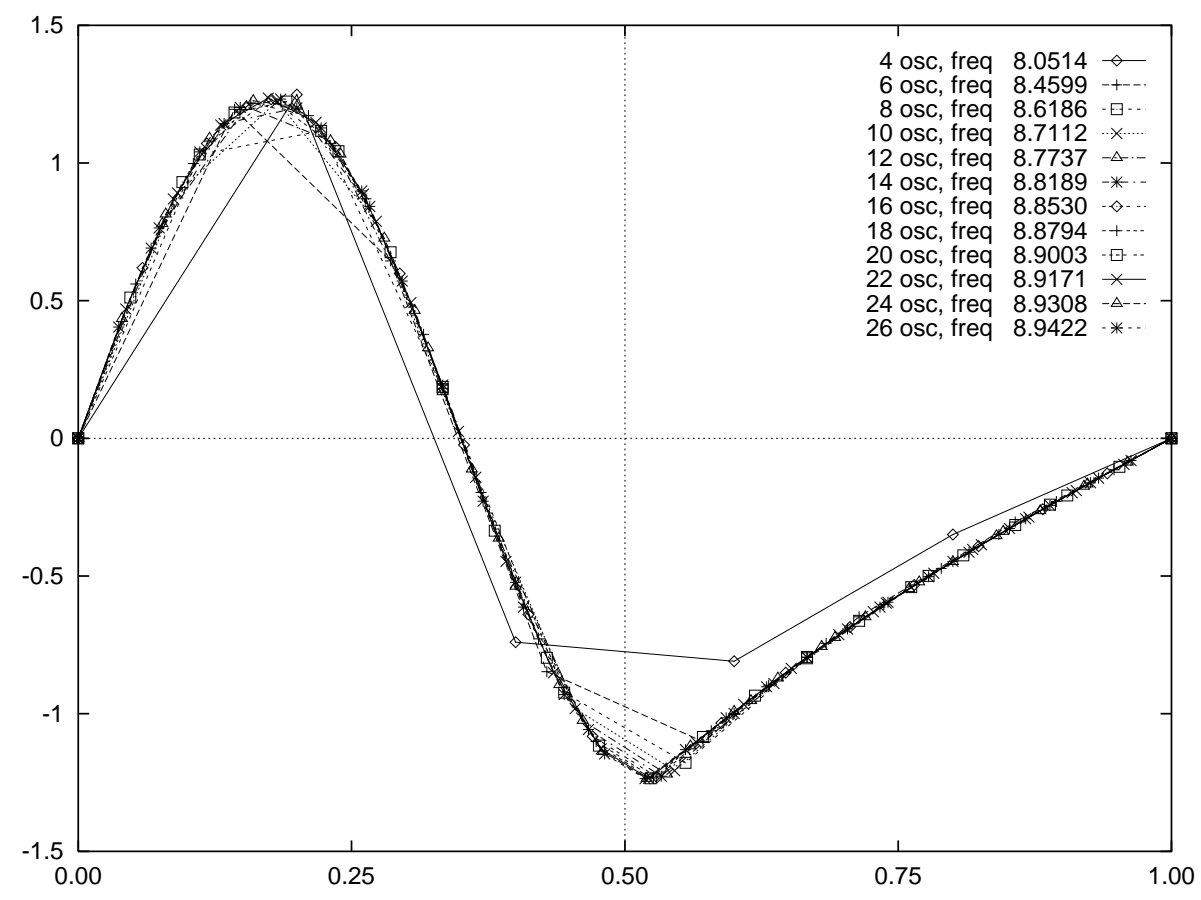

FIG. 3. The second normal mode, rescaled to the continuum normalization, for various different number of oscillators, with $L=1.0, L_{\text {in }}=0.5, \beta=0.5$.

is a sine wave in the inside region. When the mode is "abnormal" it is essentially a growing exponential. Typical modes for a small number of oscillators are shown in Figs. 1 and 2.

As $N$ becomes large, each "normal" mode and its frequency smoothly approach a limit, providing that we use a normalization appropriate for the continuum, which means that each mode must be rescaled by $\sqrt{(N+1) / L}$. See Fig. 3. As $N$ increases, each abnormal mode and its frequency undergo a smooth evolution, until at some point it disappears from the set of abnormal modes and is replaced by a normal mode with very similar form in the outside region, as shown in Fig. 1 . Because of this behavior, we believe that if we could solve the continuum behavior directly we would find just the "normal" modes.

The most striking result of the numerical solutions is that the wavenumbers of the "normal" modes are quite evenly spaced. The larger the energy, the more accurate is this approximation. In Fig. 5, we show the wavenumbers for $L=1.0, L_{\text {in }}=0.5, \beta=0.5$ which gives an energy of about 0.636. Even at this low energy the fit is good to within a few percent of the typical wavenumber. For larger energies the points will lie correspondingly closer to the line.

In the $L \rightarrow \infty$ limit, our problem has only two dimensionful parameters, $L_{\text {in }}$ and $E_{0}$. Thus there is only one dimensionless parameter, $L_{\mathrm{in}} E_{0}$, that characterizes the problem. In the 3-dimensional black hole problem the equivalent parameter is $R E_{0}$, which for the parameters in Eq. (1.3) is about $10^{13}$. Thus for application to black holes we are interested only in very large values of $L_{\mathrm{in}} E_{0}$ for which the linear approximation for the "normal" modes will be very good.

Since the "normal" mode wavenumbers extend up to $k \sim \pi$, the number of "normal" 


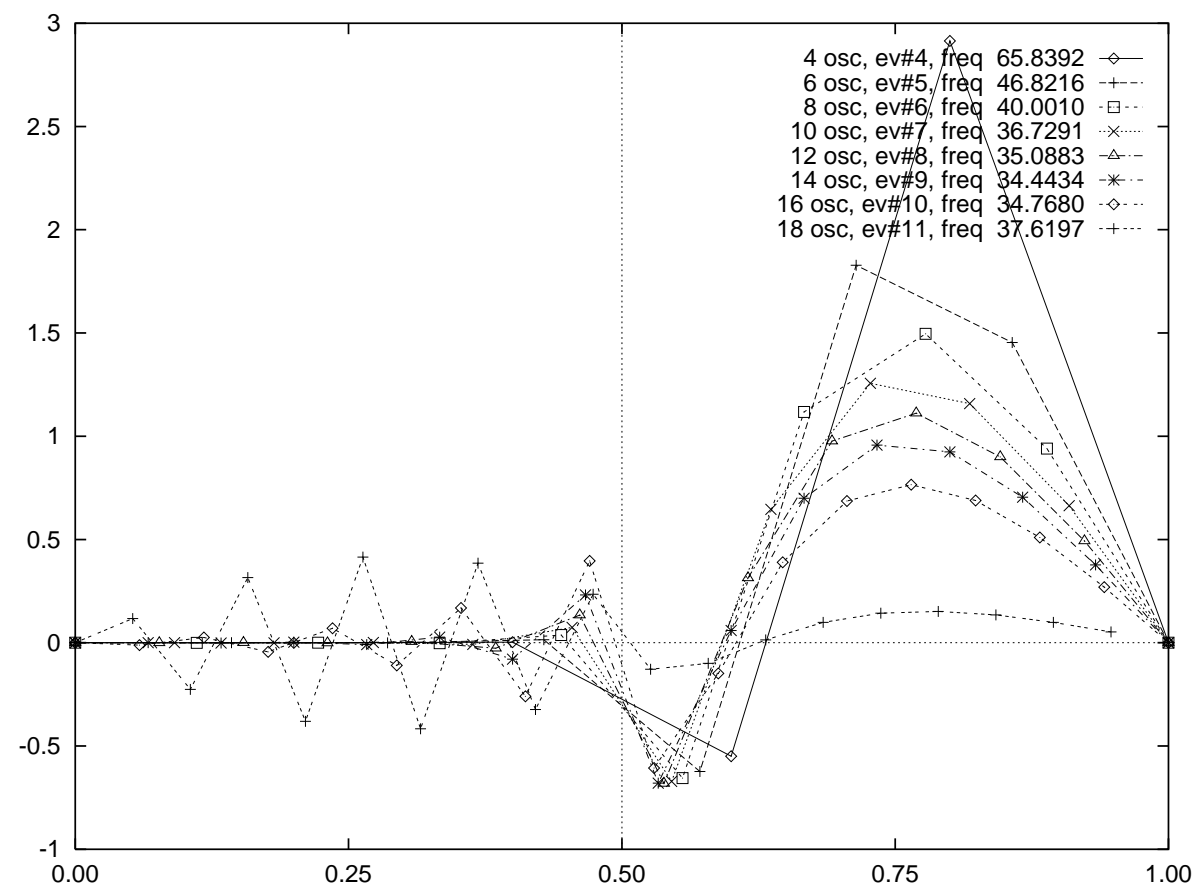

FIG. 4. An abnormal mode for $L=1.0, L_{\text {in }}=0.5, \beta=0.5$. As $N$ is increased the mode changes smoothly until at $N=18$ there is no corresponding abnormal mode, but instead one of the new normal modes has a very similar form but different normalization.

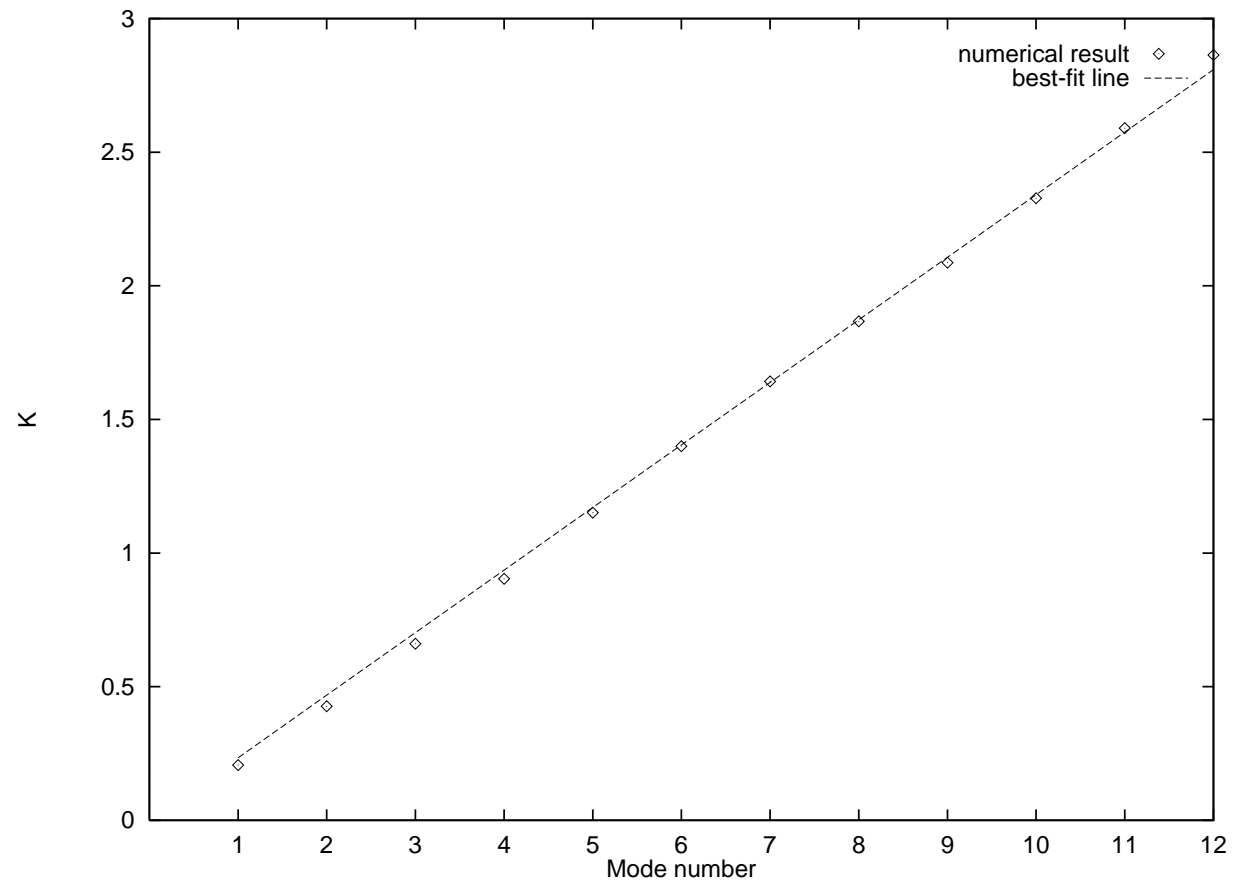

FIG. 5. The numerically-computed wavenumbers compared with the best-fit line through the origin for $L=1.0, L_{\text {in }}=0.5, \beta=0.5, N=20$. 
modes will be given an integer $N_{\text {norm }} \sim \pi / k_{1}$ where $k_{1}$ is the interval between wavenumbers. In addition there will be $N-N_{\text {norm }}$ "abnormal" modes, with frequencies $\omega>2(N+1) / L$. For $N \gg L / \beta$ these modes do not contribute to the entropy, because they are exponentially suppressed.

\section{THE FIRST OUTSIDE OSCILLATOR}

In an attempt to minimize our reliance on numerical results we will use only the statement that the wavenumbers $k_{\alpha}$ are evenly spaced. This leaves one free parameter, the spacing $k_{1}$, which depends on the energy $E_{0}$. To fix $k_{1}$ we will look at the correlator of the position of the first outside oscillator with itself, which from Eqs. (5.23a) and (6.7) is

$$
\begin{array}{r}
\left\langle x_{N_{\text {in }}+1} x_{N_{\text {in }}+1}\right\rangle=\sum_{\alpha} \frac{1}{2 \omega_{\alpha}} \operatorname{coth}\left(\frac{\beta}{2} \omega_{\alpha}\right)\left(x_{N_{\text {in }}+1}^{\alpha}\right)^{2} \\
=\sum_{\alpha} \frac{1}{2 \omega_{\alpha}} \operatorname{coth}\left(\frac{\beta}{2} \omega_{\alpha}\right) N_{\alpha}^{\prime 2} \sin ^{2} k_{\alpha}^{\prime}\left(N_{\text {in }}+1\right) .
\end{array}
$$

The important point here is that we know $x_{\mu}^{\alpha}$ for $\mu$ up to $N_{\text {in }}+1$, and we know $\left\langle x_{\mu} x_{\nu}\right\rangle$ for $\mu$ and $\nu$ down to $N_{\text {in }}+1$. Thus taking $\mu=\nu=N_{\text {in }}+1$ gives the unique correlator for which we know the components that go into the expression for the correlator while also knowing that the correlator must have the same value as in the vacuum. The same argument does not work for $\left\langle P_{N_{\text {in }}+1} P_{N_{\text {in }}+1}\right\rangle$, because we know $V_{\alpha \mu}^{-1}$ only up to $\mu=N_{\text {in }}$ and not $\mu=N_{\text {in }}+1$.

By computing this correlator in the vacuum and in a vacuum-bounded state for a given $\beta$ we can fix the spacing of the normal mode frequencies. In a high energy state, $\beta$ will be small and so there will be frequencies with $\beta \omega_{\alpha} \ll 1$. For such a frequency,

$$
\operatorname{coth} \frac{\beta}{2} \omega_{\alpha} \approx \frac{2}{\beta \omega_{\alpha}} \gg 1
$$

In order to keep $\left\langle x_{N_{\text {in }}+1}^{2}\right\rangle$ the same as in the vacuum we must require

$$
\sin ^{2} k_{\alpha}\left(N_{\text {in }}+1\right) \ll 1
$$

to cancel the large coth, and consequently

$$
k_{\alpha}\left(N_{\text {in }}+1\right) \approx n \pi
$$

for some integer $n$. This means that $k_{\alpha}$ is close to one of the wavenumbers appropriate to the problem with a rigid boundary at $L_{\text {in }}$.

\section{A. The vacuum}

First we will compute the correlator for the vacuum, $\left\langle x_{N_{\text {in }}+1}^{2}\right\rangle_{0}$. Here $\beta=\infty$ so the coth term drops out, giving us 


$$
\left\langle x_{N_{\mathrm{in}}+1}^{2}\right\rangle_{0}=\sum \frac{1}{2 \omega_{n}} N_{n}^{2} \sin ^{2} k_{n}\left(N_{\mathrm{in}}+1\right) .
$$

For the ground state of $N$ oscillators we have

$$
N_{n}^{2}=\frac{2}{N+1} \quad k_{n}=\frac{n \pi}{N+1}
$$

and

$$
\omega_{n}=2 \frac{N+1}{L} \sin \frac{k_{n}}{2}
$$

which give

$$
\left\langle x_{N_{\mathrm{in}}+1}^{2}\right\rangle_{0}=\frac{L}{2(N+1)} \sum_{n=1}^{N} \frac{\sin ^{2} n \pi \frac{N_{\mathrm{in}}+1}{N+1}}{(N+1) \sin \frac{n \pi}{2(N+1)}} .
$$

We would like to evaluate this expression in the $N \rightarrow \infty$ limit, with $N_{\text {in }} / N$ fixed. There is a prefactor $L /(N+1)$, which goes to zero in this limit, but that is just an artifact of the conventions we have used for the discrete problem, and will appear in the finite-energy vacuum-bounded states as well.

We will expand the sum for large $\mathrm{N}$. There is a logarithmic divergence. We are interested in the $\ln N$ term, and in the constant term, but we will ignore any terms of order $1 / N$ or below. Accordingly we will take $N+1$ as $N$ wherever it occurs.

First we expand the numerator using $\sin ^{2} x=(1-\cos 2 x) / 2$. The first term gives

$$
\frac{1}{2} \sum_{n=1}^{N} \frac{1}{N \sin \frac{n \pi}{2 N}}
$$

We would like to turn this sum into an integral in the $N \rightarrow \infty$ limit. However, we must first subtract out the divergent part. Accordingly, we write this as

$$
\frac{1}{\pi} \sum_{n=1}^{N} \frac{1}{n}+\frac{1}{2} \sum_{n=1}^{N}\left(\frac{1}{N \sin \frac{n \pi}{2 N}}-\frac{2}{\pi n}\right) .
$$

The first term gives

$$
\frac{1}{\pi}(\ln N+\gamma)
$$

where $\gamma$ is Euler's constant. The second term is finite and can be converted to an integral in the $N \rightarrow \infty$ limit, to give

$$
\begin{aligned}
\frac{1}{2} \int_{0}^{1} d x\left(\frac{1}{\sin \frac{\pi x}{2}}-\frac{2}{\pi x}\right) & =\frac{1}{\pi}\left[\ln \left(\frac{1}{x} \tan \frac{\pi x}{4}\right)\right]_{0}^{1} \\
& =\frac{1}{\pi} \ln \frac{4}{\pi} .
\end{aligned}
$$

The remaining term in the sum is 


$$
-\frac{1}{2} \sum_{n=1}^{N} \frac{\cos \frac{2 n \pi N_{\mathrm{in}}}{N}}{N \sin \frac{n \pi}{2 N}}
$$

To compute this we use $1 / \sin x=\csc x=1 / x+x / 6+7 x^{3} / 360+\cdots$ to get

$$
-\frac{1}{2} \sum_{n=1}^{N} \cos \frac{2 n \pi N_{\text {in }}}{N}\left(\frac{2}{\pi n}+\frac{\pi}{12} \frac{n}{N^{2}}+\frac{7 \pi}{2880} \frac{n^{3}}{N^{4}}+\cdots\right) \text {. }
$$

The first term can be summed,

$$
-\frac{1}{\pi} \sum_{1}^{\infty} \frac{\cos \frac{2 n \pi N_{\mathrm{in}}}{N}}{n}=\frac{1}{\pi} \ln \left(2 \sin \frac{\pi N_{\mathrm{in}}}{N}\right) .
$$

The rest of the terms do not contribute. Because of the oscillations of the cosine, $\sum_{1}^{N} n^{k} \cos \left(n \pi N_{\text {in }} / N\right)$ goes as $N^{k}$ rather than $N^{k+1}$ and thus is killed by the corresponding $N^{k+1}$ in the denominator.

Putting all the terms together we find that

$$
\begin{aligned}
\left\langle x_{N_{\text {in }}+1}^{2}\right\rangle_{0}=\frac{L}{2 \pi N}[\ln N & +\gamma \ln \left(\frac{8}{\pi} \sin \frac{\pi N_{\text {in }}}{N}\right) \\
& \left.+O\left(\frac{1}{N}\right)\right] .
\end{aligned}
$$

\section{B. Using the even-spacing ansatz}

Now we will compute the same correlator in the vacuum-bounded system, using the ansatz that the wavenumbers are multiples of some spacing $k_{1}$, which gives $N_{\text {norm }} \approx \pi / k_{1}$ normal wavenumbers. This is equivalent to saying that the normal wavenumbers are those appropriate to a problem with a rigid boundary at distance $L_{\text {in }}{ }^{\prime}=\left(\pi / k_{1}\right)(L /(N+1)) \approx$ $L\left(N_{\text {norm }} / N\right)$. There will also be a contribution from the abnormal modes. The contribution from each mode is positive, so by taking only normal modes we will find a lower bound on $\left\langle x_{N_{\text {in }}+1}^{2}\right\rangle$. Using this bound we can derive an upper bound on $L_{\text {in }}{ }^{\prime}$ and thus on $S(E)$.

We will again work with $N$ oscillators for $N$ large. We will ignore all terms of $O(1 / N)$ or $O\left(1 / N_{\text {norm }}\right)$. Accordingly we will take $L_{\text {in }}{ }^{\prime} / N_{\text {norm }}=L / N$ and

$$
k_{n}=n k_{1}=\frac{\pi n}{N_{\text {norm }}}=\frac{\pi n}{N_{\text {norm }}+1} .
$$

As before we have

$$
\omega_{n}=\frac{2 N}{L} \sin \frac{k_{n}}{2}=\frac{2 N}{L} \sin \frac{\pi n}{2 N_{\text {norm }}} .
$$

Now we can derive the normalizations of the normal modes: We know from Eqs. (6.10) and (6.11) that if $a$ is an inside oscillator, then for any $\mu$,

$$
\sum_{\nu} V_{a \nu} V_{\mu \nu}=\delta_{a \mu}
$$


SO

$$
\delta_{a \mu}=\sum_{n=1}^{N_{\text {norm }}} N_{n}^{2} \sin k_{n} a \sin k_{n} \mu+\text { abnormal modes. }
$$

Later we will find that $N_{\text {norm }}$ is close to $N_{\text {in }}$. Using this, and since we are working in the limit where $N_{\text {in }}$ is large, we can choose $a$ such that

$$
1 \ll a \ll 2 N_{\text {in }}-N_{\text {norm }} \sim N_{\text {in }} .
$$

Each abnormal mode $\alpha$ contributes

$$
N_{\alpha}^{2} \sinh k_{\alpha} a \sinh k_{\alpha} \mu \equiv \delta_{\mathrm{abn}}
$$

to the right-hand side of Eq. (8.21). However, this same mode contributes

$$
\frac{L}{4 N} N_{\alpha}^{2} \frac{\sinh ^{2} k_{\alpha}\left(N_{\text {in }}+1\right)}{\cosh \left(k_{\alpha} / 2\right)}
$$

to the sum for $\left\langle x_{N_{\text {in }}+1}^{2}\right\rangle$ in Eq. (8.1). Since $\left\langle x_{N_{\text {in }}+1}^{2}\right\rangle=L /(2 \pi N)[\ln N+O(1)]$ it follows that

$$
N_{\alpha}^{2} \frac{\sinh ^{2} k_{\alpha}\left(N_{\text {in }}+1\right)}{\cosh \left(k_{\alpha} / 2\right)} \lesssim \frac{\ln N}{2 \pi}
$$

and thus that

$$
\delta_{\text {abn }}<\frac{\sinh k_{\alpha} a \sinh k_{\alpha} \mu \cosh \left(k_{\alpha} / 2\right)}{2 \pi \sinh ^{2} k_{\alpha}\left(N_{\text {in }}+1\right)} \ln N .
$$

The exact values of the $k_{\alpha}$ for the abnormal modes vary with $N$. For successive $N$ values, each $k_{\alpha}$ decreases toward 0 until the corresponding mode converts to a normal mode as described in Sec. VII. By avoiding the points where these "conversions" are about to take place it is possible to find a sequence of values for $N$ which have all $k_{\alpha} \gtrsim 1$. For such values,

$$
\delta_{\mathrm{abn}} \lesssim e^{-k_{\alpha}\left(2 N_{\mathrm{in}}-a-\mu+3 / 2\right)} .
$$

From Eq. (8.22) the exponent is $\gg 1$, so $\delta_{\text {abn }}$ is exponentially small, and the contribution of the abnormal modes to (8.21) is suppressed.

Thus we ignore the abnormal modes in Eq. (8.21), multiply by $\sin k_{m} \mu$ and sum over $\mu$ to get

$$
\begin{aligned}
\sin k_{m} a & =\sum_{\mu=1}^{N_{\text {norm }}} \sin k_{m} \mu \sum_{n} N_{n}^{2} \sin k_{n} a \sin k_{n} \mu \\
& =\sum_{n} N_{n}^{2} \sin k_{n} a \sum_{\mu=1}^{N_{\text {norm }}} \sin \frac{\pi m \mu}{N_{\text {norm }}+1} \sin \frac{\pi n \mu}{N_{\text {norm }+1}} \\
& =\sum_{n} N_{n}^{2} \sin k_{n} a \cdot \frac{N_{\text {norm }}+1}{2} \delta_{m n} \\
& =N_{n}^{2} \frac{N_{\text {norm }}+1}{2} \sin k_{m} a
\end{aligned}
$$


from which we conclude that

$$
N_{n}^{2}=\frac{2}{N_{\text {norm }}+1} \approx \frac{2}{N_{\text {norm }}}
$$

Thus we have

$$
\left\langle x_{N_{\text {in }}+1}^{2}\right\rangle \gtrsim \sum_{n=1}^{N_{\text {norm }}} \frac{\sin ^{2} k_{n} N_{\text {in }}}{N_{\text {norm }} \omega_{n}} \operatorname{coth}\left(\frac{\beta}{2} \omega_{n}\right) .
$$

Now define $\Delta$ by $1-\Delta \equiv N_{\text {in }} / N_{\text {norm }} \approx L_{\text {in }} / L_{\text {in }}{ }^{\prime}$, so that $\sin ^{2} k_{n} N_{\text {in }}=\sin ^{2} n \pi(1-\Delta)=$ $\sin ^{2} n \pi \Delta$. Then put in the definition of $\omega_{n}$ from Eq. (8.19) to get

$$
\begin{aligned}
\left\langle x_{N_{\text {in }}+1}^{2}\right\rangle \gtrsim \frac{L}{2 N} & \sum_{n=1}^{N_{\text {norm }}}\left\{\frac{\sin ^{2} n \pi \Delta}{N_{\text {norm }} \sin \frac{n \pi}{2 N_{\text {norm }}}}\right. \\
& \left.\times \operatorname{coth}\left(\frac{\beta N}{L} \sin \frac{n \pi}{2 N_{\text {norm }}}\right)\right\} .
\end{aligned}
$$

In the large $N$ limit, the argument of coth becomes $\beta \pi n N /\left(2 L N_{\text {norm }}\right) \approx \beta \pi n /\left(2 L_{\text {in }}{ }^{\prime}\right)=$ $\pi n /\left(2 \tau^{\prime}\right)$ where $\tau^{\prime} \equiv L_{\text {in }}{ }^{\prime} T=L_{\text {in }}{ }^{\prime} / \beta$. Now if it were not for the coth term, we would have the same sum as in Eq. (8.8) with $N_{\text {norm }}$ for $N$ except in the prefactor. Thus we will exchange $\operatorname{coth} x$ for $1+(\operatorname{coth} x-1)=1+2 /\left(e^{2 x}-1\right)$ in Eq. (8.32) to get

$$
\begin{aligned}
\left\langle x_{N_{\mathrm{in}}+1}^{2}\right\rangle \gtrsim \frac{L}{2 \pi N}\left[\ln N_{\text {norm }}\right. & +\gamma+\ln \left(\frac{8}{\pi} \sin \pi \Delta\right) \\
& \left.+B+O\left(\frac{1}{N}\right)\right]
\end{aligned}
$$

where

$$
B \equiv \pi \sum_{n=1}^{N_{\text {norm }}} \frac{\sin ^{2} n \pi \Delta}{N_{\text {norm }} \sin \frac{n \pi}{2 N_{\text {norm }}}} \frac{2}{e^{\pi n / \tau^{\prime}}-1}
$$

Now we again use $1 / \sin x=1 / x+x / 6+\cdots$. The first term has no $N_{\text {norm }}$ dependence and we can extend the sum to $\infty$. In the next term, the sum is cut off by the exponential in the denominator, leading to a term of order $\left(\tau^{\prime} / N_{\text {norm }}\right)^{2}$. Further terms have higher powers of $\tau^{\prime} / N_{\text {norm }}$. In the limit $N_{\text {norm }} \rightarrow \infty$ we ignore all these terms, which leaves

$$
B=4 \sum_{n=1}^{\infty} \frac{\sin ^{2} n \pi \Delta}{n\left(e^{\pi n / \tau^{\prime}}-1\right)} .
$$

We are interested in the high-energy limit, for which $\tau^{\prime} \gg 1$. Later we will see that $\Delta$ is of order $\ln \tau^{\prime} / \tau^{\prime} \ll 1$. Thus the summand in Eq. (8.35) is slowly varying and we can convert the sum into an integral,

$$
B \approx 4 \int_{0}^{\infty} \frac{\sin ^{2} \pi \Delta x d x}{x\left(e^{\pi x / \tau^{\prime}}-1\right)}
$$


The error in Eq. (8.36) is approximately the term that we would have for $n=0$ in Eq. (8.35). Taking the $n \rightarrow 0$ limit we find that this term has order $\Delta^{2} \tau^{\prime} \sim\left(\ln \tau^{\prime}\right)^{2} / \tau^{\prime} \ll 1$, so our approximation is good. The integral in Eq. (8.36) can be done and the result is

$$
B=2 \pi \tau^{\prime} \Delta+\ln \frac{1-e^{-4 \pi \tau^{\prime} \Delta}}{4 \pi \tau^{\prime} \Delta} .
$$

Now we set $\left\langle x_{N_{\text {in }}+1}^{2}\right\rangle=\left\langle x_{N_{\text {in }}+1}^{2}\right\rangle_{0}$ to get

$$
\begin{array}{r}
\ln N_{\text {norm }}+\gamma+\ln \left(\frac{8}{\pi} \sin \pi \Delta\right)+B \\
\leq \ln N+\gamma+\ln \left(\frac{8}{\pi} \sin \frac{\pi N_{\text {in }}}{N}\right)
\end{array}
$$

or

$$
B \leq \ln \left(\frac{N}{N_{\text {norm }}} \frac{\sin \frac{\pi N_{\text {in }}}{N}}{\sin \pi \Delta}\right)=\ln \left(\frac{L}{L_{\text {in }}{ }^{\prime}} \frac{\sin \frac{\pi L_{\text {in }}}{L}}{\sin \pi \Delta}\right) .
$$

Note that we have taken the limit $N \rightarrow \infty$ and there is no longer any dependence on $N$. If we now let $L \rightarrow \infty$ with $L_{\text {in }}$ fixed, we can approximate $\sin \left(\pi L_{\text {in }} / L\right)=\pi L_{\text {in }} / L$ to get

$$
B \leq \ln \frac{L_{\text {in }}}{L_{\text {in }}{ }^{\prime} \sin \pi \Delta}=\ln \frac{1-\Delta}{\sin \pi \Delta} \approx \ln \frac{1}{\pi \Delta}
$$

since $\Delta$ is small.

Using $B$ from Eq. (8.37) we get

$$
2 \pi \tau^{\prime} \Delta+\ln \frac{1-e^{-4 \pi \tau^{\prime} \Delta}}{4 \pi \tau^{\prime} \Delta} \leq \ln \frac{1}{\pi \Delta} .
$$

Thus $\Delta \leq \Delta_{\max }$ where

$$
\Delta_{\max }=\frac{1}{2 \pi \tau^{\prime}} \ln \frac{4 \tau^{\prime}}{1-e^{-4 \pi \tau^{\prime} \Delta_{\max }}} .
$$

If instead of $\tau^{\prime}=L_{\text {in }}{ }^{\prime} T$ we use $\tau \equiv L_{\text {in }} T$ we will make an error of order $\Delta_{\text {max }}$, which we expect to be small. So we ignore the second order contribution and take $\tau^{\prime}$ as $\tau$ in Eq. (8.42),

$$
\Delta_{\max }=\frac{1}{2 \pi \tau} \ln \frac{4 \tau}{1-e^{-4 \pi \tau \Delta_{\max }}} .
$$

If we ignore $e^{-4 \pi \tau \Delta_{\max }}$ in the denominator we get

$$
\Delta_{\max }=\frac{1}{2 \pi \tau} \ln 4 \tau \text {. }
$$

Using this we find that $e^{-4 \pi \tau \Delta_{\max }}=(4 \tau)^{-2} \ll 1$ since $\tau \gg 1$, which justifies ignoring this term. We will also ignore $\ln 4$ by comparison with $\ln \tau$. Thus we conclude

$$
\Delta \lesssim \frac{1}{2 \pi \tau} \ln \tau+O\left(\frac{1}{\tau}\right)=\frac{1}{2 \pi L_{\mathrm{in}} T} \ln L_{\mathrm{in}} T+O\left(\frac{1}{L_{\mathrm{in}} T}\right)
$$

and

$$
L_{\text {in }}{ }^{\prime} \leq L_{\text {in }}+\frac{1}{2 \pi T} \ln L_{\text {in }} T+O\left(\frac{1}{T}\right) .
$$

The equivalent system is larger by at most a thermal wavelength times a logarithmic factor depending on the inside size. 


\section{PROPAGATION OF BOUNDS}

In the previous section we derived an expression that gives the frequencies, and thus the entropy, for a vacuum-bounded system at a given temperature $T=1 / \beta$. Given such an expression, we would like to compute the entropy as a function of energy. But the energy is not simple to compute from the frequencies alone.10 However, we can easily compare the entropy of the vacuum-bounded system to that of a system with a rigid boundary at $L_{\text {in }}$ and the same temperature. To make this comparison at fixed energy instead, we proceed as follows.

Consider the free energy $F=E-T S$ which has $d F=-S d T$. Integrating gives

$$
E-T S=-\int_{0}^{T} S\left(T^{\prime}\right) d T^{\prime}
$$

Let $S^{\mathrm{rb}}, T^{\mathrm{rb}}$ denote the entropy and temperature of the system with a rigid boundary at $L_{\text {in }}$, and $S, T$, and so on denote those for the vacuum bounded system. For any quantity $A$ let $\delta A(T)$ denote the difference between vacuum-bounded and rigid-bounded systems at fixed temperature, $\delta A(T) \equiv A(T)-A^{\mathrm{rb}}(T)$, and $\delta A(E)$ denote the same difference at fixed energy, $\delta A(E) \equiv A(E)-A^{\mathrm{rb}}(E)$. With $E$ fixed we compare the differences (to first order) in the two sides of Eq. (9.1) between the vacuum-bounded and rigid-bounded systems,

$$
-T \delta S(E)-\delta T(E) S=-\delta T(E) S-\int_{0}^{T} \delta S\left(T^{\prime}\right) d T^{\prime}
$$

where the first term on the right-hand side comes from the change in the integration limit. Thus

$$
\delta S(E)=\frac{1}{T} \int_{0}^{T} \delta S\left(T^{\prime}\right) d T^{\prime}
$$

\section{DERIVING THE BOUND}

Now we apply these results to the case from Sec. VIIIB where

$$
\omega_{n} \approx \frac{n \pi}{L_{\mathrm{in}}{ }^{\prime}} \quad \text { and } \quad L_{\mathrm{in}}{ }^{\prime} \approx L_{\mathrm{in}}(1+\Delta)
$$

with

$$
\Delta L_{\mathrm{in}} \leq \frac{1}{2 \pi T} \ln L_{\mathrm{in}} T
$$

\footnotetext{
${ }^{10}$ It can be done, but since $E$ needs to be renormalized against the ground-state energy of the entire system the result depends sensitively on the frequencies and normalizations even for very high-energy modes.
} 
This says that at any given temperature, the vacuum-bounded system has the entropy $S(T)$ of a system of length $L_{\text {in }}{ }^{\prime}$. Now in a one-dimensional system the entropy density is proportional to the temperature,

$$
S^{\mathrm{rb}}=\frac{\pi}{3} L_{\mathrm{in}} T
$$

and thus the entropy difference between vacuum-bounded and rigid-bounded systems is

$$
\delta S(T)=\frac{\pi}{3} \Delta L_{\text {in }} T \leq \frac{1}{6} \ln L_{\text {in }} T .
$$

Using the results from Sec. 【X,

$$
\delta S(E) \leq \frac{1}{T} \int_{0}^{T} \frac{1}{6} \ln L_{\mathrm{in}} T^{\prime} d T^{\prime}=\frac{1}{6}\left(\ln L_{\mathrm{in}} T-1\right) .
$$

Since we are ignoring terms of order 1 by comparison with those of order $\ln T$, we can write this 11

$$
\delta S(E) \lesssim \frac{1}{6} \ln L_{\text {in }} T .
$$

So, we conclude that the vacuum-bounded condition closely approximates the rigid box of length $L_{\text {in }}$. For the same energy, the vacuum-bounded condition allows slightly more entropy. The entropy difference grows at most logarithmically with the number of thermal wavelengths in the inside region. Since the total entropy at a given temperature is linear in $T$, the ratio of $\delta S$ to $S$ goes to zero at high temperatures.

\section{DISCUSSION}

We have introduced a new way of specifying that matter and energy are confined to a particular region of space. Rather than giving a boundary condition per se, we specify a condition on a density matrix describing the state of the overall system. We require that any measurement which does not look into the inside region cannot distinguish our system from the vacuum. This avoids certain difficulties such as the Casimir energy that results from the introduction of a boundary and the geometric entropy [23,24] that results from ignoring part of a system. For these "vacuum-bounded" states, we consider the problem of finding the maximum-entropy state for a given total energy. This is analogous to the problem of finding the thermal state in a system with a rigid boundary.

Unfortunately, the vacuum-bounded problem is more difficult than the analogous problem with a rigid boundary and we must resort to working in one dimension and to numerical solution on a lattice. From the numerical solution we justify the ansatz that the continuum

\footnotetext{
${ }^{11}$ It has happened that $\delta S(E)$ and $\delta S(T)$ are approximately the same, but that is a particular property of the system at hand. For example, if $\Delta$ were a constant we would have $\delta S(E)=$ $1 / T \int_{0}^{T} c \Delta L_{\mathrm{in}} T^{\prime} d T^{\prime}=2 c \Delta L_{\mathrm{in}} T=2 \delta S(T)$.
} 
wavenumbers are evenly spaced in this problem. Using this ansatz we compute an upper bound on the entropy of a vacuum-bounded state, and show that for high energies $(E R \gg 1)$ the entropy approaches that of a system with rigid boundaries. Of course this is what one would expect for a system whose typical wavelengths are much shorter than the size of the inside region.

To apply this result to an evaporating black hole we look at the state produced by the black hole after evaporation [3]. Since our calculation was one-dimensional we must assume that the similarity between the vacuum-bounded state and the thermal state with a rigid boundary extends to 3 dimensions. Then we infer that very little entropy can be emitted in the final explosion, confirming the results of Aharonov, Casher and Nussinov [17 and Preskill [3]. For example, a black-hole formed in the big bang with mass of order $10^{15} \mathrm{~g}$ would be evaporating today. During its life it would have radiated entropy $S \sim 10^{38}$. Now we assume that the entropy of the final explosion has energy $E \sim 10^{19} \mathrm{erg}$ contained in radius $R \sim 10^{-23} \mathrm{~cm}$ as in Sec. $[\mathrm{B}$, and that the maximum entropy is not too different from that of a spherical box, in accord with our 1-dimensional result. Then we find that the final explosion can emit only entropy $S \sim 10^{10}$, which is a factor of $10^{28}$ too little to produce a pure state.

This argument means that either a black hole must not evaporate completely but rather leave a remnant or remnants, that information must be lost, or else that the Hawking radiation is not exactly thermal, even at very early times [12].

\section{ACKNOWLEDGMENTS}

We would like to thank Alan Guth and Sean Carroll for much advice and assistance; Niklas Dellby, Eddie Farhi, Dan Freedman, Ken Halpern, Andy Latto, Arthur Lue, Keith Ramsay and Peter Unrau for helpful conversations; Harlequin Inc. for providing a copy of their LispWorks product on which some of the computations were done; Bruno Haible and Marcus Daniels for their CLISP Common Lisp implementation; and Kevin Broughan and William Press for making available a version of Numerical Recipes translated into LISP 12.

This work is supported in part by funds provided by the U.S. Department of Energy (D.O.E.) under cooperative research agreement DE-FC02-94ER40818.

\section{APPENDIX A: CONCAVITY OF THE ENTROPY}

Lieb [25] showed that $S$ is always (downward) concave. Here we obtain the same result by a different technique and show also that the concavity is strict, i.e. that $d^{2} S / d t^{2}$ never vanishes.

We consider $S=S\left(\rho^{\prime}\right)=-\operatorname{Tr} \rho^{\prime} \ln \rho^{\prime}$ with $\rho^{\prime} \equiv \rho+t \delta \rho$ for some $\delta \rho$ with $\operatorname{Tr} \delta \rho=0$. Then

$$
\frac{d S}{d t}=-\operatorname{Tr} \delta \rho \ln \rho^{\prime}
$$

\footnotetext{
${ }^{12}$ These routines and many other are now available on a Numerical Recipes CDROM [27]
} 
and

$$
\frac{d^{2} S}{d t^{2}}=-\operatorname{Tr} \delta \rho \frac{d}{d t} \ln \rho^{\prime}
$$

To expand this we use the formula

$$
\frac{d}{d t} \ln A=\int_{0}^{1} d s(I-s(A-I))^{-1} \frac{d A}{d t}(I-s(A-I))^{-1}
$$

which can easily be derived as follows: Let $B=A-I$. We expand

$$
\ln A=\ln (I+B)=B+\frac{B^{2}}{2}+\frac{B^{3}}{3}+\cdots
$$

and differentiate to get

$$
\begin{aligned}
\frac{d}{d t} \ln A & =\frac{d B}{d t} \frac{1}{2}\left(\frac{d B}{d t} B+B \frac{d B}{d t}\right) \\
+ & \frac{1}{3}\left(\frac{d B}{d t} B^{2}+B \frac{d B}{d t} B+B^{2} \frac{d B}{d t}\right)+\cdots
\end{aligned}
$$

We observe that this is

$$
\begin{array}{rl}
\int_{0}^{1} & d s \frac{d B}{d t}+s\left(\frac{d B}{d t} B+B \frac{d B}{d t}\right)+s^{2}\left(\frac{d B}{d t} B^{2}+B \frac{d B}{d t} B+B^{2} \frac{d B}{d t}\right) \\
& =\int_{0}^{1} d s\left(1+s B+s^{2} B^{2}+\cdots\right) \frac{d B}{d t}\left(1+s B+s^{2} B^{2}+\cdots\right) \\
& =\int_{0}^{1} d s(I-s B)^{-1} \frac{d B}{d t}(I-s B)^{-1}
\end{array}
$$

as desired.

Using Eq. (A3) we find

$$
\begin{aligned}
\frac{d^{2} S}{d t^{2}} & =-\int_{0}^{1} d s \operatorname{Tr} \delta \rho(I-s(\rho-I))^{-1} \delta \rho(I-s(\rho-I))^{-1} \\
& =-\int_{0}^{1} \operatorname{Tr} X(s)^{2}
\end{aligned}
$$

where

$$
X(s) \equiv(\delta \rho)^{1 / 2}(I-s(\rho-I))^{-1}(\delta \rho)^{1 / 2} .
$$

Since $X(s)$ is Hermitian, $\operatorname{Tr} X(s)^{2} \geq 0$ with equality obtained only for $X(s)=0$. Thus

$$
\frac{d S^{2}}{d t^{2}} \leq 0
$$

with equality only if $X(0)=0$ for all $s$. But $X(0)=\delta \rho$, and thus

$$
\frac{d S^{2}}{d t^{2}}<0
$$

for any nonzero $\delta \rho$. 


\section{REFERENCES}

[1] S. Hawking, Phys. Rev. D 248, 30 (1974).

[2] D. N. Page, in Proceedings of the 5th Canadian Conference on General Relativity and Relativistic Astrophysics, edited by R. Mann and R. McLenaghan (World Scientific, River Edge, NJ, 1994), pp. 1-41.

[3] J. Preskill, in International Symposium on Black Holes, Membranes, Wormholes, and Superstrings (World Scientific, River Edge, NJ, 1993), pp. 22-39.

[4] T. Banks, in String Theory, Gauge Theory, and Quantum Gravity, edited by R. Dijgraaf, K. Narain, and S. Randjbar-Daemi (North-Holland, Amsterdam, 1995), pp. 21-65.

[5] S. W. Hawking, Phys. Rev. D 53, 3099 (1996).

[6] S. B. Giddings, Phys. Rev. D 49, 4078 (1994).

[7] T. Banks, L. Susskind, and M. E. Peskin, Nucl. Phys. B244, 125 (1984).

[8] A. Strominger, preprint USCSBTH-94-34, Unitary Rules for Black Hole Evaporation, hep-th/9410187.

[9] A. Strominger and J. Polchinski, Phys. Rev. D 50, 7403 (1994).

[10] L. Susskind and J. Uglum, in String theory, gauge theory and quantum gravity: proceedings of the Trieste Spring School and Workshop, ICTP, Trieste, Italy, 27 March-7 April, 1995, edited by R. Dijkgraaf (North-Holland, Amsterdam, 1996).

[11] Y. Kiem, H. Verlinde, and E. Verlinde, Phys. Rev. D 52, 7053 (1995).

[12] E. Keski-Vakkuri, G. Lifschytz, S. D. Mathur, and M. E. Ortiz, Phys. Rev. D 51, 1764 (1995).

[13] S. Bose, L. Parker, and Y. Peleg, Phys. Rev. D 54, no. 10 (1996), to appear.

[14] A. Casher et al., preprint TAUP-2344-96, Black hole horizon fluctuations, hep-th/9606106.

[15] A. Strominger and C. Vafa, Phys. Rev. Lett. B379, 99 (1996).

[16] E. Keski-Vakkuri and P. Kraus, preprint CALT-68-2079, hep-th/9610045, and references therein.

[17] Y. Aharonov, A. Casher, and S. Nussinov, Phys. Lett. B 191, 51 (1987).

[18] F. Wilczek, in International Symposium on Black Holes, Membranes, Wormholes, and Superstrings (World Scientific, River Edge, NJ, 1993), pp. 1-21.

[19] J. D. Bekenstein, in General Relativity: proceedings of the 7th Marcel Grossman Meeting, edited by R. Ruffini and M. Keiser (World Scientific, River Edge, NJ, 1995).

[20] J. D. Bekenstein, Phys. Rev. D 27, 2262 (1983).

[21] W. G. Unruh and R. M. Wald, Phys. Rev. D 27, 2271 (1983).

[22] D. N. Page, Phys. Rev. D 13, 198 (1976).

[23] M. Srednicki, Phys. Rev. Lett. 71, 666 (1993).

[24] C. Callan and F. Wilczek, Phys. Lett. B 333, 55 (1994).

[25] E. H. Lieb, Bull. Am. Math. Soc. 81, 1 (1975).

[26] M. J. D. Powell, in Numerical Methods for Nonlinear Algebraic Equations, edited by P. Rabinowitz (Gordon and Breach, London, New York, 1969), Chap. 7, pp. 87-114.

[27] W. H. Press, S. A. Teukolsky, W. T. Vetterling, and B. P. Flannery, Numerical Recipes: The Art of Scientific Computing, Code CDROM v 2.06 (Cambridge University Press, Cambridge, 1996). 